\title{
Metallic Nanowires by Full Wafer Stencil Lithography
}

\author{
0. Vazquez-Mena, ${ }^{*}$ G. Villanueva, V. Savu, K. Sidler, M. A. F. van den Boogaart, \\ and J. Brugger*
}

Microsystems Laboratory, Ecole Polytechnique Federale de Lausanne (EPFL),
1015 Lausanne, Switzerland

Received June 20, 2008; Revised Manuscript Received August 11, 2008

\begin{abstract}
Aluminum and gold nanowires were fabricated using $100 \mathrm{~mm}$ stencil wafers containing nanoslits fabricated with a focused ion beam. The stencils were aligned and the nanowires deposited on a substrate with predefined electrical pads. The morphology and resistivity of the wires were studied. Nanowires down to $70 \mathrm{~nm}$ wide and $5 \mu \mathrm{m}$ long have been achieved showing a resistivity of $10 \mu \Omega \mathrm{cm}$ for Al and $5 \mu \Omega \mathrm{cm}$ for $\mathrm{Au}$ and maximum current density of $\sim 10^{8} \mathrm{~A} / \mathrm{cm}^{2}$. This proves the capability of stencil lithography for the fabrication of metallic nanowires on a full wafer scale.
\end{abstract}

An important objective in nanotechnology is the development of alternative nanopatterning methods and the fabrication of novel nanoscale structures and materials. Among such structures, nanowires (NWs) have shown potential and applications in a broad range of fields such as electronics, ${ }^{1,2}$ magnetic memories, ${ }^{3}$ thermoelectric, ${ }^{4,5}$ nanomechanical, ${ }^{6}$ optoelectronic, ${ }^{7}$ and biosensing devices ${ }^{8-10}$ due to their physical properties and surface to volume ratio. In particular, metallic nanowires can be applied for interconnects, magnetic memories based on spin-polarized current ${ }^{3}$ and biosensors. ${ }^{9}$ To fabricate NWs, the two approaches used are the chemical synthesis (bottom-up) and the nanopatterning methods (topdown). ${ }^{1}$ The common techniques for top-down nanopatterning are electron beam lithography (EBL) ${ }^{11}$ focused ion beam (FIB), ${ }^{12}$ deep-UV lithography (DUV), ${ }^{13}$ and nanoimprint lithography (NIL). ${ }^{14,15}$ EBL and FIB have a very high resolution $(<10 \mathrm{~nm})$ but they are serial techniques, limiting their throughput. DUV is a well-established parallel technique developed for silicon-based technologies with high throughput and largely used in semiconductor industry, but it relies on complex equipment and processing rules. NIL is a parallel patterning technique with high throughput and resolution $(\sim 10 \mathrm{~nm})$ that requires embossing a resist layer with a stamp. Since EBL, DUV, and NIL are resist-based techniques, they require resist coating, chemical solvents, heat treatments, energy radiation or pressure embossing (NIL) on the substrate; this limits their application on substrates with high topography, fragile, or with materials that can be damaged by energy radiation or pressure. An alternative method for nanopatterning is stencil lithography (SL). SL is a shadow

* To whom correspondence should be addressed. E-mail: (O.V.M.) oscar.vazquez@epfl.ch; (J.B.) juergen.brugger@epfl.ch. masking technique, illustrated in Figure 1a, that only requires to put a stencil onto a substrate for direct and parallel pattering by deposition, ${ }^{16}$ etching, ${ }^{17}$ or implantation ${ }^{18}$ of the substrate through the stencil apertures without any resist processing. Compared to the previous techniques, SL has a lower resolution and the patterning area is limited by the size and stability of the membranes; nevertheless, SL has been used to pattern metallic dots $<50 \mathrm{~nm}$ in diameter with chip size stencils ${ }^{16,19}$ and areas up to $1 \times 3 \mathrm{~mm}^{2}$ have been patterned with $300 \mathrm{~nm}$ metallic dots. ${ }^{20}$ Since SL does not require any resist processing, it has the advantage of reducing the number of steps required for patterning and allowing the patterning of a broad range of substrates compared to resistbased techniques. For instance, SL has been used to deposit metals,${ }^{20}$ fullerenes,${ }^{21}$ organic conductive molecules,${ }^{22}$ complex oxides ${ }^{23}$ and magnetic alloys ${ }^{24}$ and to pattern different substrates such as self-assembled monolayers (SAMs), ${ }^{25}$ organic layers, ${ }^{26}$ polymer substrates, ${ }^{27}$ CMOS devices, ${ }^{28}$ cantilevers, and nonplanar substrates. ${ }^{20}$ Another important advantage of SL is that the stencils can be reused many times. ${ }^{19,26,29,30}$ In particular stencils containing nanoapertures have been used up to 12 times for $\mathrm{Al}$ depositions without showing any degradation or damage on the membranes. ${ }^{29}$ The fabrication of stencils with silicon nitride membranes is based on conventional silicon microfabrication techniques. Once the stencil has been fabricated, it makes the patterning of metals simpler than using resist-based methods such as lift-off or metal etching. In Table 1 we compare the steps required for metal patterning using a stencil, lift-off, and etching.

In this letter, we present the parallel fabrication of metallic NWs on full wafer scale (100 mm diameter) using stencil 

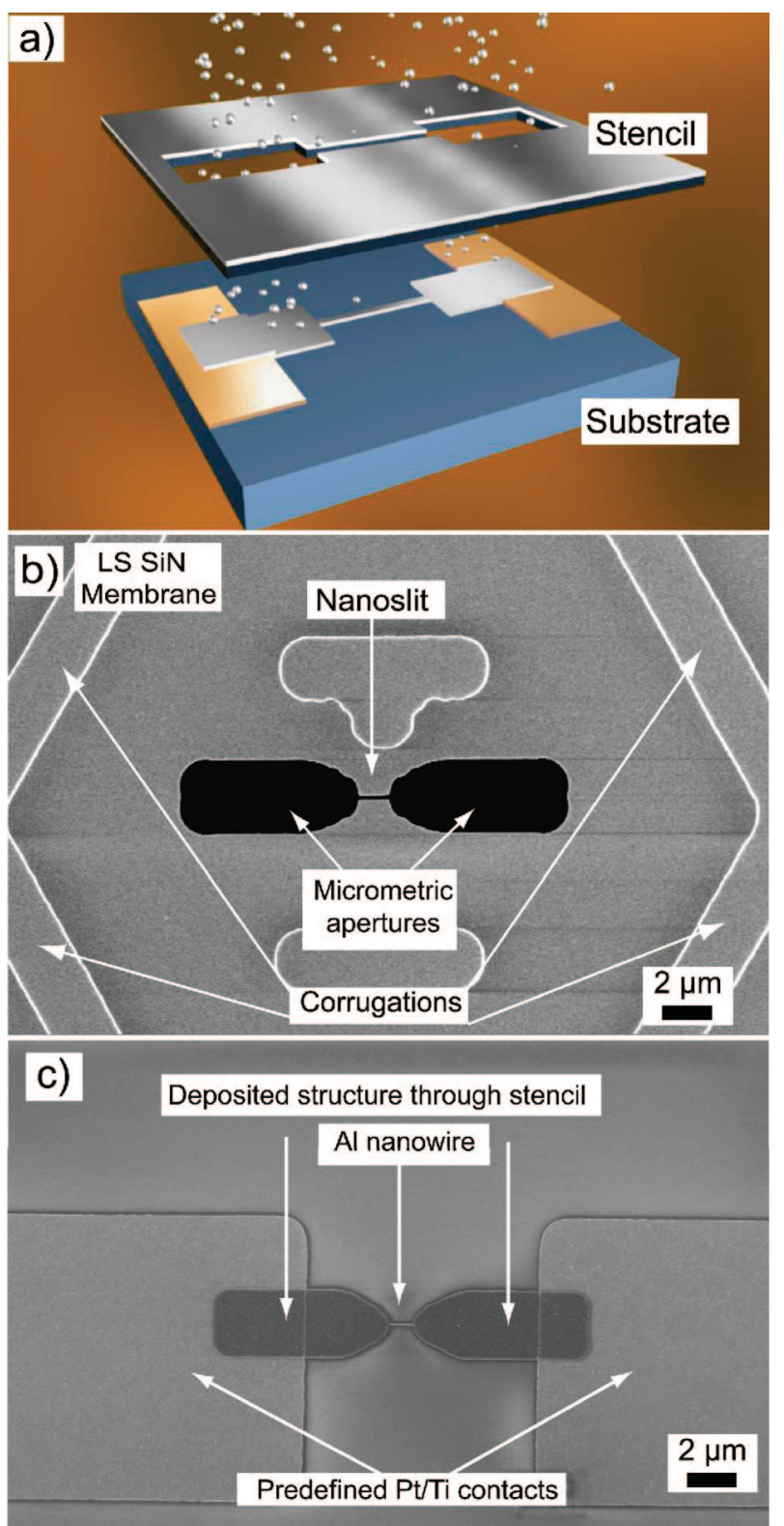

Figure 1. Fabrication of nanowires by stencil lithography. (a) Schematics showing the principle of SL for NW deposition. The stencil is put and aligned on top of a substrate. Then an evaporated material passes through the stencil apertures forming NWs onto a prepatterned substrate without any resist, etching, or lift-off processing. (b) Stencil membrane made of low-stress silicon nitride (LS SiN) containing a nanoslit in between two side micrometric apertures. The membrane is reinforced using corrugations (see Supporting Information, Figure SI 1 and 2). (c) Aluminum structure deposited through a stencil. It consists of a NW in between two side micrometric structures corresponding to the aperture in the stencil. This structure is deposited in between predefined contacts to allow electrical measurements.

lithography. In this work, we have analyzed the dimensions, the electrical resistivity, and the maximal current density before breaking of the nanowires. The stencils used for this work are made of thin reinforced membranes containing $<100 \mathrm{~nm}$ wide nanoslits fabricated by focused ion beam (FIB) milling. These stencils have been used to deposit $\mathrm{Al}$ and Au NWs with nominal thicknesses of 60 and $45 \mathrm{~nm}$, respectively, and widths in the range from 65 to $175 \mathrm{~nm}$ on
Table 1. Comparison of Steps Required for Metal Patterning

\begin{tabular}{|c|c|c|}
\hline stencil & lift-off & etching \\
\hline metal deposition & $\begin{array}{l}\text { resist coating } \\
\text { alignment and } \\
\text { exposure } \\
\text { resist development } \\
\text { metal deposition } \\
\text { resist lift-off }\end{array}$ & $\begin{array}{l}\text { metal deposition } \\
\text { resist coating } \\
\text { alignment and } \\
\text { exposure } \\
\text { resist development } \\
\text { metal etching } \\
\text { resist removal }\end{array}$ \\
\hline
\end{tabular}

substrates containing predefined electrical contact pads. The width and thickness of the deposited NWs were analyzed in order to study the pattern transfer from the apertures on the stencil to the deposited structures. This study revealed a size enlargement with respect to the stencil aperture, a thickness reduction compared to the nominal deposited thickness, and a polycrystalline structure of the NWs. The NWs show an ohmic behavior with an electrical resistivity higher than bulk values and a maximal current density in the order of $10^{8}$ $\mathrm{A} / \mathrm{cm}^{2}$.

The stencils fabricated for this work contain corrugated membranes with nanoslits patterned by FIB milling and distributed across the entire wafer. ${ }^{31,32}$ The membranes are made of $100 \mathrm{~nm}$ thick low stress silicon nitride (LS SiN) with dimensions of $100 \mu \mathrm{m}$ in width and $1 \mathrm{~mm}$ in length. This membrane thickness is required in order to pattern sub$100 \mathrm{~nm}$ apertures; however, membranes with such thickness are fragile to physical stress and may suffer deformations and ruptures. ${ }^{19,33}$ To increase their stability, the fabricated membranes are corrugated instead of being planar. The corrugations have a hexagonal ring geometry shown in Figure $1 \mathrm{~b}$. These corrugations give more stability to the membrane by increasing its moment of inertia. ${ }^{31,33}$ The stencil apertures are shown in Figure 1b. They consist of two side micrometric apertures $\left(\sim 5 \times 3 \mu \mathrm{m}^{2}\right)$ defined by UV lithography $(0.8 \mu \mathrm{m}$ resolution) with a nanoslit in between them fabricated by FIB milling. The width of the nanoslits is defined by the FIB whereas the length corresponds to the distance between the two side micrometric apertures. The stencils also contain alignment apertures that should match with their corresponding alignment marks on the substrate. Two stencils were fabricated, one for $\mathrm{Al}$ deposition and the other for $\mathrm{Au}$ deposition. The stencil used for Al deposition contains nanoslits with lengths $L=1,2$, and $5 \mu \mathrm{m}$ and the one for Au deposition has nanoslits with $L=0.8,1.6$, and $4.6 \mu \mathrm{m}$. In both cases, the nanoslits have widths $\left(W_{\mathrm{St}}\right)$ from 50 to $140 \mathrm{~nm}$. (Stencil fabrication process, corrugations, and a full wafer stencil are shown in the Supporting Information, Figures SI 1 and SI 2.)

$\mathrm{Al}$ and $\mathrm{Au}$ NWs were deposited on substrates with a 200 $\mathrm{nm}$ thick silicon oxide layer, predefined electrical contact pads, and alignment marks. In the substrate for the deposition of $\mathrm{Al} \mathrm{NWs,} \mathrm{the} \mathrm{contacts} \mathrm{pads} \mathrm{and} \mathrm{the} \mathrm{alignment} \mathrm{marks} \mathrm{were}$ made of a film of $\operatorname{Pt}(45 \mathrm{~nm}) / \mathrm{Ti}(5 \mathrm{~nm})$. On the substrate for Au NWs, they were made of $\mathrm{Au}(40 \mathrm{~nm}) / \mathrm{Ti}(5 \mathrm{~nm})$. These structures were prepared by UV lithography, metal deposition, and lift-off process. To deposit the NWs, the stencils were aligned and clamped with their respective substrate using a standard bond aligner system (Suss MA6/BA6). Then 


\section{Stencil apertures}
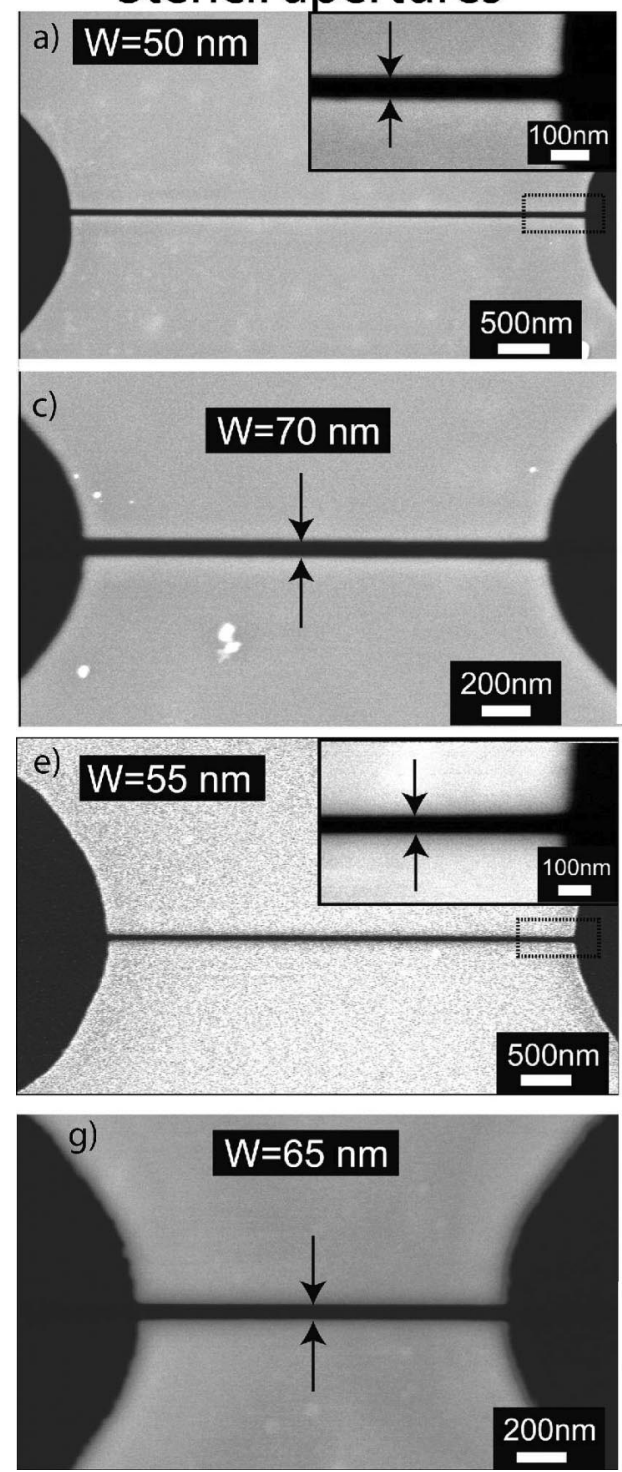

\section{Deposited nanowires}
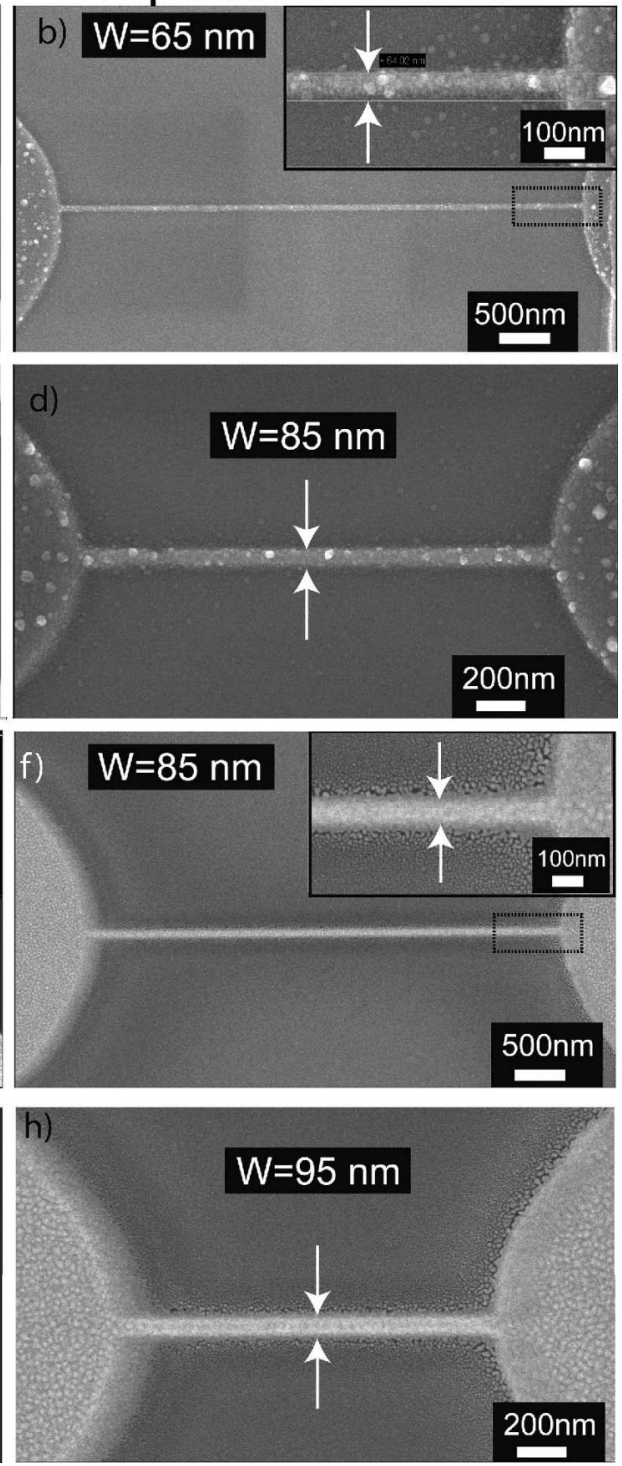

Figure 2. Stencil apertures and NWs deposited by SL. The stencil aperture patterns are clearly transferred to the substrate forming NWs with two side micrometric structures. (a) Stencil aperture $(L=5 \mu \mathrm{m}, W=50 \mathrm{~nm})$ and (b) corresponding Al NW $65 \mathrm{~nm}$ wide. (c) Stencil aperture $(L=2 \mu \mathrm{m}, W=70 \mathrm{~nm})$ and (d) corresponding Al NW $85 \mathrm{~nm}$ wide. (e) Stencil aperture $(L=4.6 \mu \mathrm{m}, W=55 \mathrm{~nm})$ and (f) corresponding Au NW $85 \mathrm{~nm}$ wide. (g) Stencil aperture $(L=1.6 \mu \mathrm{m}, W=65 \mathrm{~nm})$ and (h) corresponding Au NW $95 \mathrm{~nm}$ wide.

the stencil and substrate clamped for $\mathrm{Al}$ deposition were mounted into an evaporator for the deposition of $60 \mathrm{~nm}$ thick Al by e-beam evaporation. The same process was used for the stencil and substrate for $\mathrm{Au}$, depositing $45 \mathrm{~nm}$ thick $\mathrm{Au}$ by e-beam evaporation as well. In both cases, the depositions were done at room temperature without any temperature controller, base pressure of $10^{-6} \mathrm{mbar}$, and a deposition rate of $0.4 \mathrm{~nm} / \mathrm{s}$. The distance from the source to the substrate was $1 \mathrm{~m}$, and the material source $\sim 1 \mathrm{~cm}$ in diameter. The deposited structures consist of two side micrometric structures (to facilitate electrical contact) with a NW in between, corresponding to the apertures in the stencil membranes as illustrated in Figure 1c. The NWs are deposited in between the predefined electrical contact pads to allow electrical measurements. Due to the curvature of the stencil and the substrate, there is a gap between them during the metal deposition. For full wafer stencils and substrates $100 \mathrm{~mm}$ in diameter, the size of the gap varies between 10 and $20 \mu \mathrm{m}$ across the stencil-substrate interface (measured from focal distances with an optical microscope). This limits the resolution compared to chip-size $(\sim 1 \mathrm{~cm})$ stencil deposition where there is a reduced and more uniform gap size $(\sim 1$ $\mu \mathrm{m})$ between stencil and substrate.

Figure 2 illustrates deposited $\mathrm{Al}$ and $\mathrm{Au}$ NWs with their corresponding stencil apertures. The NWs were aligned with a lateral precision of $\sim 1 \mu \mathrm{m}$. The length of the NWs corresponds to the length of the nanoslits in the stencils. For the $\mathrm{Al}$ NWs the width ( $W_{\mathrm{NW}}$ ) ranges from 65 to $160 \mathrm{~nm}$ and for the Au NWs $W_{\mathrm{NW}}$ ranges from 80 to $175 \mathrm{~nm}$ as measured by scanning electron microscopy (SEM). In both cases, $\mathrm{Au}$ and $\mathrm{Al}$, the pattern in the stencil aperture is clearly transferred as a metallic NW on the substrate. The NWs were analyzed by SEM and atomic force microscopy (AFM) and compared with their respective stencil apertures in order to study the 
pattern transfer from the stencil apertures to the deposited structures. ${ }^{34}$ This analysis revealed that the NWs are wider than the nanoslits by a few tens of nanometers. The thickness of the NWs is also affected by the width of the stencil apertures; below a critical size of stencil apertures, the thickness of the NWs decreases. The AFM and SEM analysis also show that the NWs have a polycrystalline structure.

In Figure 3a the width of the NWs $\left(W_{\mathrm{NW}}\right)$ is plotted as a function of the stencil aperture width $\left(W_{\mathrm{St}}\right)$, showing a linear trend for both $\mathrm{Al}$ and Au NWs. After fitting the data to a linear function, we obtain $W_{\mathrm{Al}-\mathrm{NW}}=1.03 W_{\mathrm{St}}+11.81 \mathrm{~nm}$ for $\mathrm{Al}$ and $W_{\mathrm{Au}-\mathrm{NW}}=1.11 W_{\mathrm{St}}+26.77 \mathrm{~nm}$ for Au. From these expressions and for this size range, we can make a simple model of the width of NWs deposited by stencil lithography fixing the slope of $W_{\mathrm{NW}}$ versus $W_{\mathrm{St}}$ to $1: W_{\mathrm{NW}}=$ $W_{\mathrm{St}}+\mathrm{SE}$, where SE is the size enlargement of the NWs with respect to the stencil apertures. For Al NWs SE $=15.00$ $\pm 3.93 \mathrm{~nm}$ and for Au NWs SE $=35.86 \pm 5.93 \mathrm{~nm}$. The analysis by tapping mode AFM shows that the thickness of the NWs $\left(t_{\mathrm{NW}}\right)$ depends on the width of the stencil apertures. Figure $3 \mathrm{~b}$ shows the AFM image of an Al NW (same as in Figure $2 \mathrm{~b}$ ) from where we have extracted cross sections through one of the micrometric side structures (CS-CT) and through the NW (CS-NW). The cross sections are shown in Figure 3c. This clearly shows that the micrometric side structure has the expected nominal thickness $t_{\mathrm{n}-\mathrm{Al}}=60 \mathrm{~nm}$, defined from the $\mathrm{Al}$ deposition, whereas the NW thickness is only $30 \mathrm{~nm}$. This behavior is observed also for Au NWs (Supporting Information, Figure SI 3). In Figure 3e, we have plotted $t_{\mathrm{NW}}$ as a function of the aperture width $W_{\mathrm{St}}$. For $W_{\mathrm{St}}$ $\sim 130 \mathrm{~nm}$, the NWs have the nominal thickness of $t_{\mathrm{n}-\mathrm{Al}}=$ $60 \mathrm{~nm}$ for $\mathrm{Al}$ and $t_{\mathrm{n}-\mathrm{Au}}=45 \mathrm{~nm}$ for Au. However, for $W_{\mathrm{St}}<$ $130 \mathrm{~nm}$ the thickness of the deposited structures decreases. For the narrowest apertures of $W_{\mathrm{St}} \sim 50 \mathrm{~nm}$, the deposited $\mathrm{Al}$ and $\mathrm{Au}$ NWs have a thickness close to half of the nominal value ( $20 \mathrm{~nm}$ for $\mathrm{Au}$ and $30 \mathrm{~nm}$ for $\mathrm{Al}$ ). In the case of the $\mathrm{Au}$ NWs, the reduction follows a linear trend, whereas in the case of $\mathrm{Al} \mathrm{NWs}$ the values are more dispersed but still the reducing trend is clear. The SEM and AFM images also revealed a polycrystalline structure for both $\mathrm{Al}$ and $\mathrm{Au}$ NWs. From the SEM images we estimate a grain size in the 20-30 $\mathrm{nm}$ range for both $\mathrm{Al}$ and $\mathrm{Au} \mathrm{NWs}$ without any observable dependence on their width or thickness. The rms roughness of the $\mathrm{Al} \mathrm{NWs}$ is $1.8 \mathrm{~nm}$ whereas for $\mathrm{Au}$ is $0.52 \mathrm{~nm}$ (grain size and roughness shown in the Supporting Information, Figure SI 4). The blurring of the structures is observed in Figure $3 \mathrm{~d}$, forming a thin layer of material $<5 \mathrm{~nm}$ and some isolated grains around the nanowire. The same behavior has been observed for Au NWs (see Supporting Information, Figure SI 4c). This blurred zone is extended $\sim 100-200 \mathrm{~nm}$ on each side of the NWs. The blurring is a consequence of the existence of a gap between the stencil and substrate as reported previously on structures deposited by stencil lithography. ${ }^{16,33,35-37}$

In order to extract the resistivity of the nanowires, electrical DC measurements at room temperature $(\sim 293 \mathrm{~K})$ were performed using a probe station and a HP parameter analyzer.
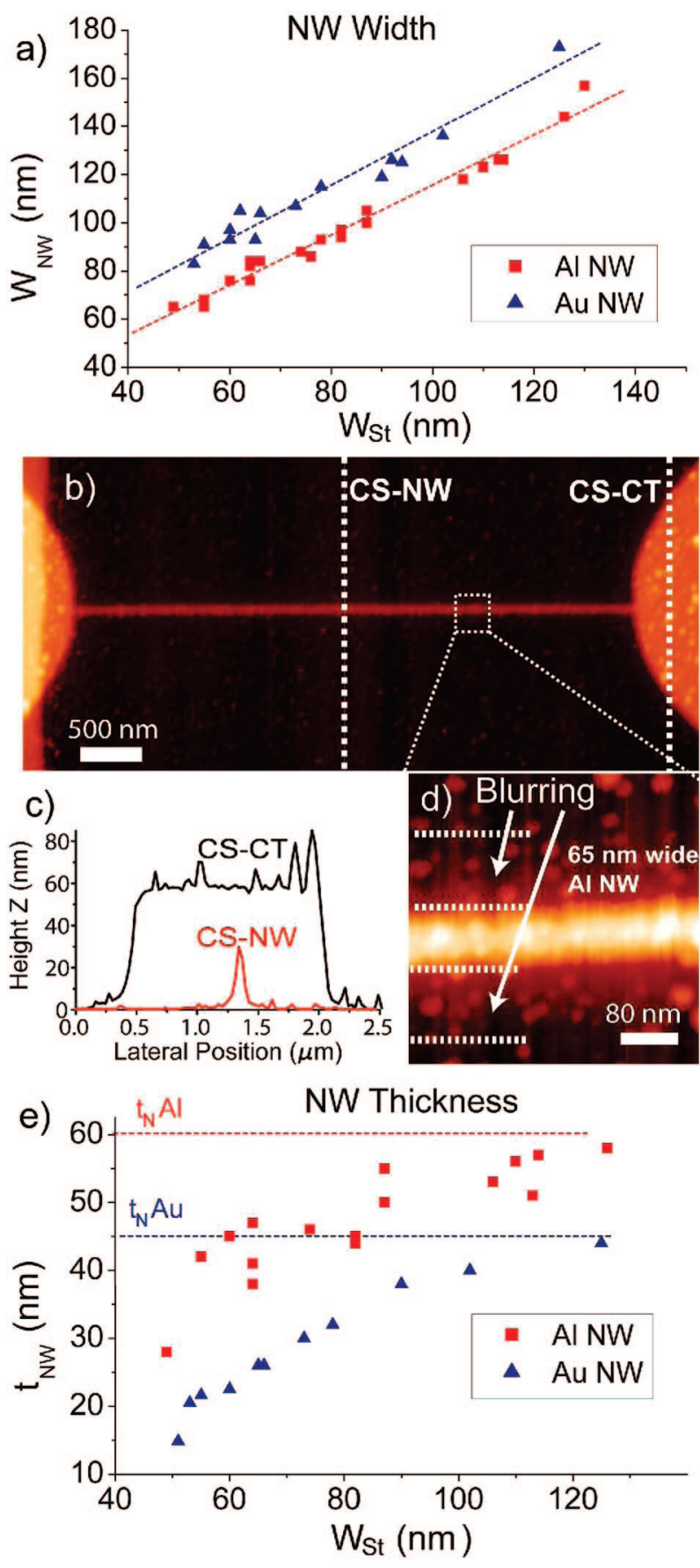

Figure 3. (a) Width of $\mathrm{Al}$ and $\mathrm{Au} \mathrm{NWs}\left(W_{\mathrm{NW}}\right)$ distributed on the full wafer as a function of the width of the nanoslits in the stencils $\left(W_{\mathrm{St}}\right)$. The $\mathrm{Al} \mathrm{NWs}$ are wider than the stencil slits by $W_{\mathrm{NW}}-W_{\mathrm{St}}=15 \pm 4 \mathrm{~nm}$, whereas the Au NWs by $35 \pm 5 \mathrm{~nm}$. (b) Tapping Mode AFM image of an Al NW showing two cross section paths. CS-CT goes across one of the side micrometric structures and CS-NW goes across the NW. (c) Cross section profiles of CS-CT and CS-NW in panel c showing the difference in thickness between the micrometric structure and the NW (CSCT, $60 \mathrm{~nm}$; CS-NW, $30 \mathrm{~nm}$ ). (d) Zoom into the NW with $W_{\mathrm{NW}}$ $=65 \mathrm{~nm}$. The arrows show the blurring surrounding the NW. This blurred part is $<5 \mathrm{~nm}$ thick and also has isolated grains. (e) NW thickness $t_{\mathrm{NW}}$ as a function of stencil aperture width $W_{\mathrm{St}}$. The values of $t_{\mathrm{N}-\mathrm{Al}}=60 \mathrm{~nm}$ and $t_{\mathrm{N}-\mathrm{Au}}=45 \mathrm{~nm}$ refer to the nominal deposited thickness of $\mathrm{Al}$ and $\mathrm{Au}$, respectively. The thickness of the NWs is reduced from the nominal thickness for $W_{\mathrm{St}}<130 \mathrm{~nm}$. 

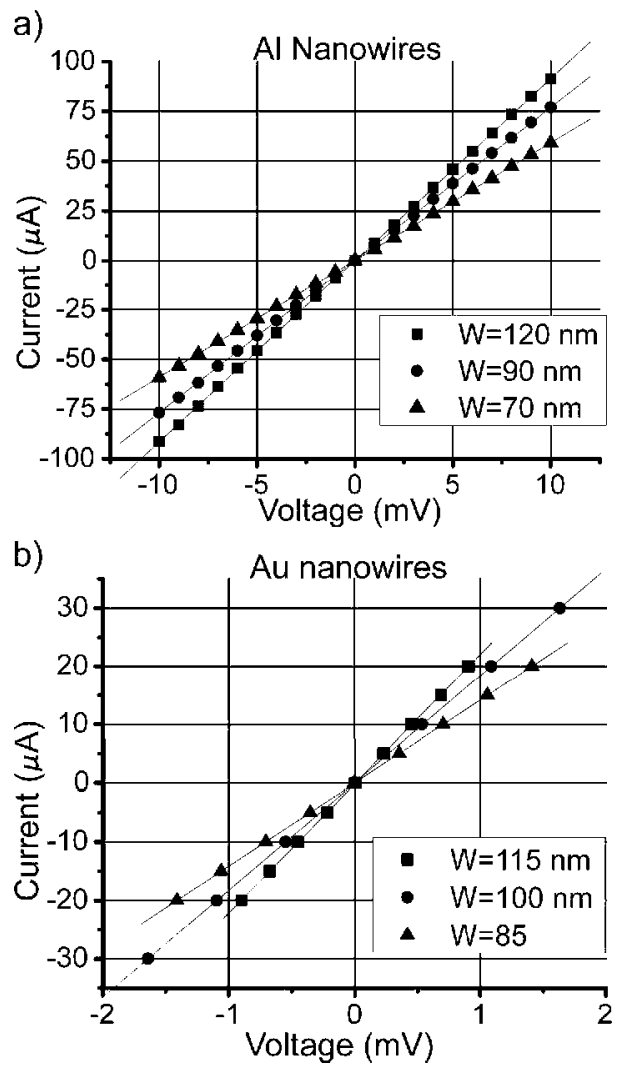

Figure 4. Current vs voltage curves. Top: $I-V$ curves for Al NWs with $L=2 \mu \mathrm{m}$ and widths of 120,90 , and $70 \mathrm{~nm}$. The NWs show an ohmic behavior and their corresponding resistances are 115,125 , and $160 \Omega$, respectively. These values include the contact resistance $(\sim 70 \Omega$ ). Bottom: $I-V$ curves for Au NWs with $L=1.6 \mu \mathrm{m}$ and widths of 115,100 , and $85 \mathrm{~nm}$ wide showing also an ohmic behavior. Their respective resistances are 45,55 , and $70 \Omega$. These values include the contact resistance $(\sim 20 \Omega)$. In both cases, $\mathrm{Au}$ and $\mathrm{Al}$, the resistance increases as the width of the NWs decreases. (Measured NWs shown in the Supporting Information, Figure SI 5).

The resistance of the nanowires was measured keeping the electrical current below $100 \mu \mathrm{A}$ to prevent wire breakdown. In Figure 4 the current versus voltage $(I-V)$ curves for three $\mathrm{Al}$ NWs and three Au NWs are shown (measured NWs shown in the Supporting Information, Figure SI 5). The measurements show the characteristic linear behavior for metallic structures. In a few NWs, we applied larger current levels and we observed an ohmic behavior up to $\sim 500 \mu \mathrm{A}$ and for larger currents the NWs eventually broke down as shown in Figure 6. The resistance $R$ of the NWs increases as the width is decreased, as expected from the resistance of a conductor $R=\rho L / A$ (eq 1 ) where $\rho$ corresponds to the resistivity of the material and $A$ is the cross section area. The measured resistance consist of the resistance from the NWs plus the contact resistance from the predefined contact pads $\left(R_{\text {Measured }}=R_{\mathrm{NW}}+R_{\text {Contact }}\right)$. The contact resistance was estimated by the linear fitting of the $R_{\text {Measured }}$ versus $A^{-1}$ relation $\left(R_{\text {Measured }}=\alpha A^{-1}+\beta\right)$, and identifying $\alpha \mathrm{A}^{-1}$ as the nanowire resistance and $\beta$ as the contact resistance. For the Al NWs, we have estimated a contact resistance from the $\mathrm{Pt} / \mathrm{Ti}$ predefined contact pads of $70 \pm 7 \Omega$ and for the $\mathrm{Au}$ NW pads a contact resistance of $20 \pm 5 \Omega$ from the $\mathrm{Au} / \mathrm{Ti}$
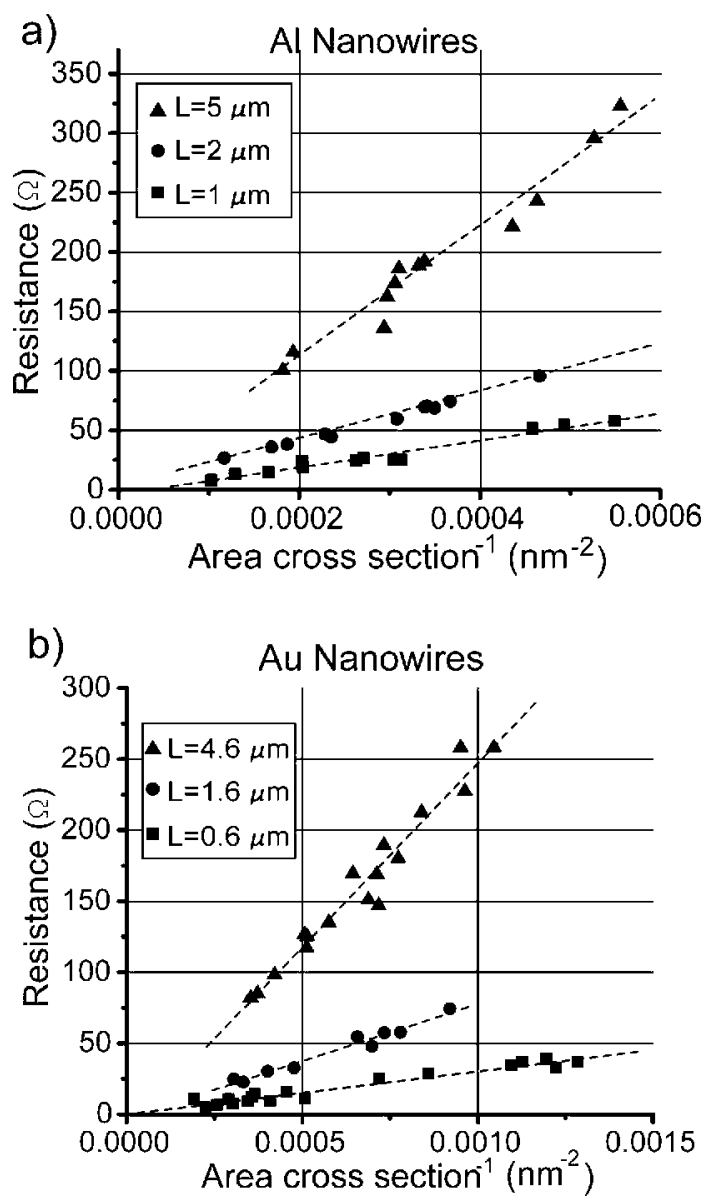

Figure 5. Resistance of $\mathrm{Al}$ (top) and $\mathrm{Au}$ (down) NWs distributed on the full wafer as a function of the inverse of the area cross section $\left(A^{-1}\right)$ for different wire lengths. The resistance increases as the area cross section decreases. The slope of the curves is also proportional to the length of the NWs. Resistivities for Al: $L=5 \mu \mathrm{m}, \rho=11$ $\mu \Omega \mathrm{cm} ; L=2 \mu \mathrm{m}, \rho=9.27 \mu \Omega \mathrm{cm} ; L=1 \mu \mathrm{m}, \rho=11.3 \mu \Omega \mathrm{cm}$. Resistivities for $\mathrm{Au}: L=4.6 \mu \mathrm{m}, \rho=5.43 \mu \Omega \mathrm{cm} ; L=1.6 \mu \mathrm{m}, \rho$ $=5.02 \mu \Omega \mathrm{cm} ; L=0.6 \mu \mathrm{m}, \rho=5.03 \mu \Omega \mathrm{cm}$.

pads. This contact resistance was subtracted for each measured resistance to obtain the resistance from the NWs. In Figure 5 the resistance of the $\mathrm{Al}$ and $\mathrm{Au}$ NWs distributed on the full wafer is plotted as a function of $A^{-1}$ for different lengths. The cross section area $(A)$ was calculated from the width and thickness measured from SEM and AFM images respectively. As observed in Figure 5, the resistance of the NWs increases as $A$ is reduced and the slope of the curves is proportional to the length of the wire as expected from eq 1. To extract the resistivity of the NWs, we have used the slope of $R$ versus $A^{-1}$ from Figure 5, $\mathrm{d} R / \mathrm{d} A^{-1}=\rho L$, since the length of the NWs is known. Taking the average from the slopes of the different lengths of NWs, the resistivity found for $\mathrm{Al}$ is $\rho_{\mathrm{Al}-\mathrm{NW}}=10.5 \pm 1.09 \mu \Omega \mathrm{cm}$ and for $\mathrm{Au}$ $\rho_{\mathrm{Au}-\mathrm{NW}}=5.16 \pm 0.2 \mu \Omega \mathrm{cm}$. As a reference, we also deposited test films of $\mathrm{Al}$ and $\mathrm{Au}$ during the same evaporation as the NWs $\left(t_{\mathrm{Au}}=45 \mathrm{~nm}\right.$ and $\left.t_{\mathrm{Al}}=60 \mathrm{~nm}\right)$. The resistivity of these test films was $\rho_{\mathrm{Al} ; \mathrm{t}=60 \mathrm{~nm}}=8 \mu \Omega \mathrm{cm}$ and $\rho_{\mathrm{Au}: t=45 \mathrm{~nm}}=5 \mu \Omega \mathrm{cm}$. The behavior of the NWs was explored at higher voltages (voltage ramp rate of $200 \mathrm{mV} / \mathrm{sec}$, steps of $20 \mathrm{mV}$ ). A nonlinear behavior was observed for current 

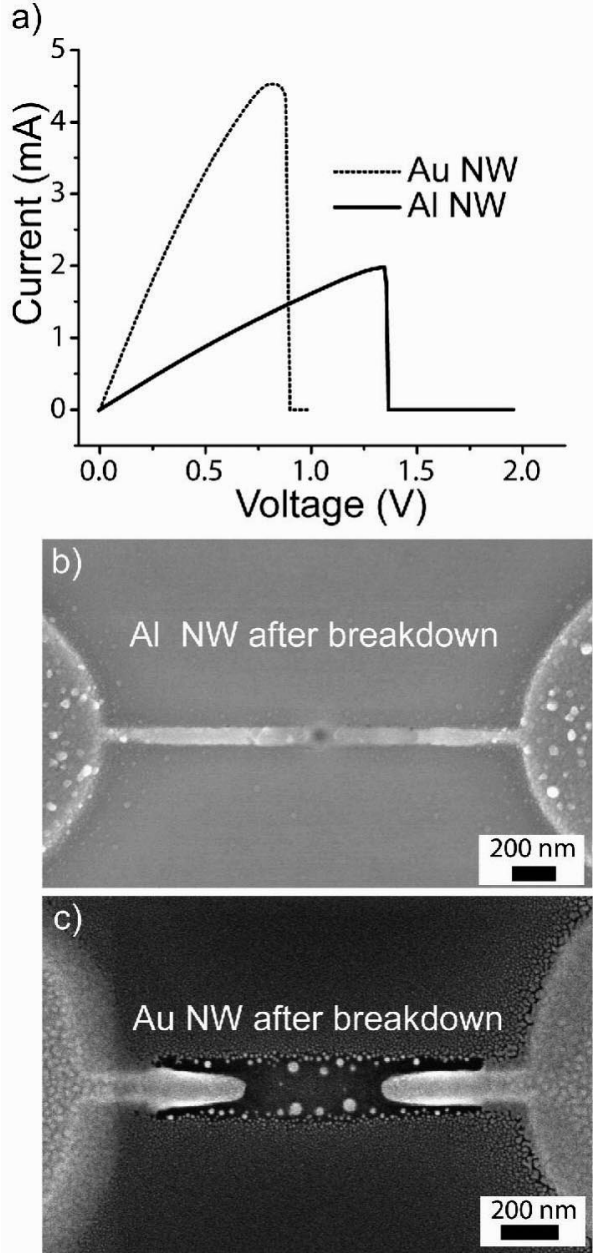

Figure 6. $I$ vs $V$ for Au and Al nanowires at large voltages (voltage ramp rate of $200 \mathrm{mV} / \mathrm{sec}$ ). (a) The measured $\mathrm{Au} \mathrm{NW}$ has dimensions $W_{\mathrm{NW}}=100 \mathrm{~nm}, t=30 \mathrm{~nm}$ and $L=1.6 \mu \mathrm{m}$. The Au NW starts to show a nonlinear behavior at a current density of $J=$ $7 \times 10^{7} \mathrm{~A} / \mathrm{cm}^{2}(I=2 \mathrm{~mA})$, and it breaks down at a current density of $J_{\max }=1.5 \times 10^{8} \mathrm{~A} / \mathrm{cm}^{2}$ as observed from the drastic current drop at $I=4.5 \mathrm{~mA}$ and $V=0.8 \mathrm{~V}$. In the case of Al, the dimensions of the NW are $W_{\mathrm{NW}}=65 \mathrm{~nm}, t=28 \mathrm{~nm}$ and $L=2 \mu \mathrm{m}$. The nonlinear behavior starts at a $J=5 \times 10^{7} \mathrm{~A} / \mathrm{cm}^{2}(I=1 \mathrm{~mA})$ and the breakdown of the wires is at $J_{\max }=1 \times 10^{8} \mathrm{~A} / \mathrm{cm}^{2}$ as the current drastically drops at $I=2 \mathrm{~mA}$ and $V=1.3 \mathrm{~V}$. (b,c) Images of the $\mathrm{Al}$ and $\mathrm{Au}$ NWs after breakdown

densities $J>5 \times 10^{7} \mathrm{~A} / \mathrm{cm}^{2}$. Above this value, the NWs show an increase in resistivity until they breakdown. We measured 6 Au NWs obtaining an average maximal current density of $J_{\max }=1.76 \pm 0.45 \times 10^{8} \mathrm{~A} / \mathrm{cm}^{2}$ and $3 \mathrm{Al} \mathrm{NWs}$ with $J_{\max }=1.12 \pm 0.18 \times 10^{8} \mathrm{~A} / \mathrm{cm}^{2}$. Figure 6 illustrates the case of an $\mathrm{Al}$ and a Au NW. For the Au NW (100 nm wide), the maximal current density $\left(J_{\max }\right)$ before breaking yields $1.5 \times 10^{8} \mathrm{~A} / \mathrm{cm}^{2}$. For the $\mathrm{Al} \mathrm{NW}$ ( $65 \mathrm{~nm}$ wide), it breaks down at $J_{\max }=1.1 \times 10^{8} \mathrm{~A} / \mathrm{cm}^{2}$. The failure normally occurred in the middle of the NWs, where they probably reach the highest temperature.

The resolution of stencil lithography is limited due to the inherent gap between stencil and substrate during deposition. This gap produces two effects: first, the material coming from the source to the substrate is deposited not only under the stencil aperture but also underneath the membrane as

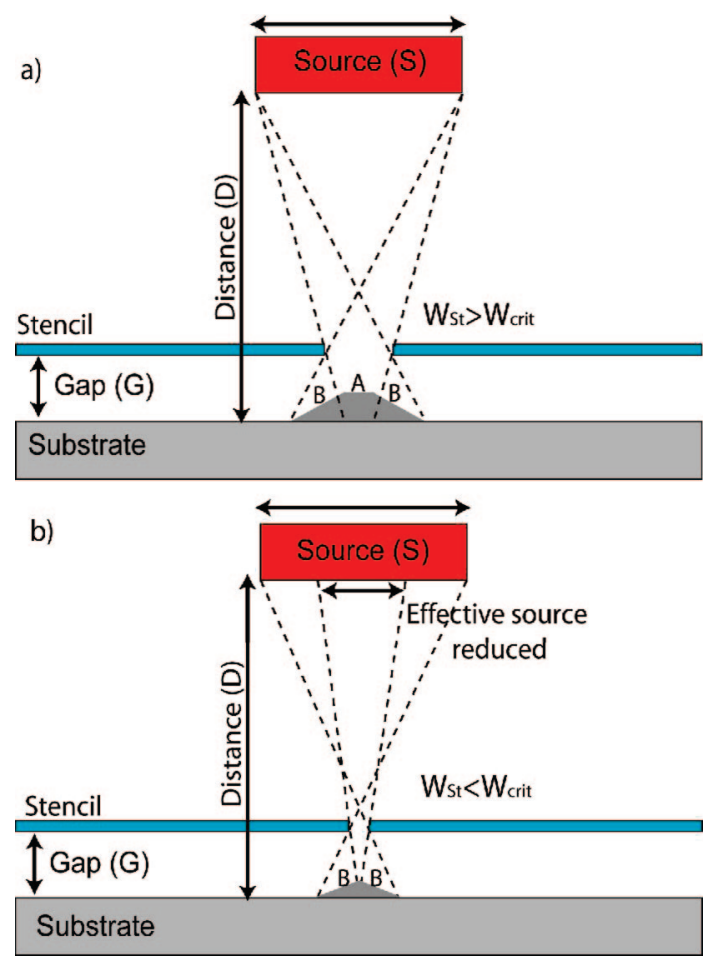

Figure 7. Stencil aperture and effective source size. (a) For aperture widths $W_{\mathrm{St}}$ larger than a critical width $W_{\text {Crit }}$, there is a zone (A) on the substrate under the aperture that receives material from the entire source and there is a side zone (B) that receives material only from a fraction of the source so it is thinner than zone (A). (b) For aperture widths $W_{\mathrm{St}}$ smaller than a critical aperture $W_{\text {Crit }}$, then there is not such a zone $(\mathrm{A})$ on the substrate receiving material from the entire source. All the points on the substrate receive material only from a fraction of the source, reducing the source effective size. As consequence, the structures are thinner since there is less material arriving to the substrate. From geometrical considerations $W_{\text {Crit }}=$ $G S / D$. (Image is not in scale and surface diffusion of material is not illustrated).

illustrated in Figure 7; and second, once the material lands on the surface, due to surface diffusion, the material is able to spread since there are no lateral physical barriers preventing the material from going beyond the area under the stencil aperture. These two effects produce a structure in the substrate that is larger than the stencil aperture. The size enlargement, the blurring, and the reduced thickness of the NWs are a consequence of the existence of this stencilsubstrate gap. The final size and profile of the structures is determined by several factors like the source-stencil-substrate configuration (gap size, material source size, and sourcesubstrate distance), ${ }^{33}$ the clogging of the aperture, ${ }^{33}$ substrate properties ${ }^{30}$ surface diffusion (temperature), ${ }^{37}$ and the scattering through the stencil aperture. ${ }^{19}$ The reduced thickness of the deposited NWs is a consequence of the reduction in the effective size of the material source when the stencil apertures are smaller than a critical size. This effect is illustrated in Figure 7. If the aperture width of the stencil $W_{\mathrm{St}}$ is larger than a critical aperture $W_{\text {Crit, }}$ then we can identify two zones (A) and (B) on the substrate. The central zone (A) receives material emitted from the entire source where as the side zone (B) receives material only from a fraction of the source. As a consequence, the zone (B) is thinner than 
zone (A). However, if $W_{\text {St }}$ is smaller than $W_{\text {Crit }}$, then there is no such zone (A) and instead all the points on the substrate receive material only from a certain fraction of the source. This reduces the amount of material arriving to the substrate, hence reducing the thickness of the deposited structure. From geometrical considerations we find $W_{\text {Crit }}=(G)(S) /(D)$, where $G$ is the stencil-substrate gap distance, $S$ the size of the material source, and $D$ the distance from source to substrate. Using this expression with $W_{\text {Crit }}=130 \mathrm{~nm}$ (critical size extracted from Figure $3 \mathrm{e}), D=1 \mathrm{~m}$ and $S=1 \mathrm{~cm}$, we can estimate a gap distance of $G \sim 13 \mu \mathrm{m}$, which is in the range for the typical gap between stencil and substrate $(10-20 \mu \mathrm{m})$.

The measured room-temperature resistivity of the NWs $\left(\rho_{\mathrm{Al}-\mathrm{NW}}=10.5 \mu \Omega \mathrm{cm}\right.$ and $\left.\rho_{\mathrm{Au}-\mathrm{NW}}=5.16 \mu \Omega \mathrm{cm}\right)$ is larger than the reported bulk resistivity of $\mathrm{Al}$ and $\mathrm{Au}\left(\rho_{\mathrm{Al}-\text { bulk }}=\right.$ $2.65 \mu \Omega \mathrm{cm}$ and $\rho_{\text {Au-bulk }}=2.21 \mu \Omega \mathrm{cm}$ at $\left.293 \mathrm{~K}\right) .{ }^{38}$ It is known that the conductivity of metallic films and wires decreases when the size scales are comparable to or smaller than the electron mean free path $(\mathrm{Al} \sim 15 \mathrm{~nm}$ and $\mathrm{Au} \sim 40$ $\mathrm{nm}$ at room temperature ${ }^{39}$ ) due mainly to two phenomena: (1) scattering at the surfaces ${ }^{40,41}$ and (2) scattering at grain boundaries of conduction electrons. ${ }^{42}$ The effect of these factors on the electrical resistivity depends on the degree of specularity of the scattering at the surface and on the electron reflectivity at the grain boundaries of the nanowires. Comparing the resistivity of the NWs with the resistivity of the test thin films and with the resistivity of the bulk, we found that for $\mathrm{Al}$ the NWs resistivity is only 1.3 times larger than the Al test film ( $t=60 \mathrm{~nm}$ ) resistivity but 3.9 times larger than $\mathrm{Al}$ bulk value. In the case of $\mathrm{Au}$ NWs we found that $\rho_{\mathrm{Au}-\mathrm{NW}}$ is 1.22 times larger than $\rho_{\mathrm{Au}-\mathrm{t}}=45 \mathrm{~nm}$ but 2.3 times larger than $\rho_{\text {Au-bulk. }}$ Similar results have been obtained previously by Durkan et al. ${ }^{43}$ for $\mathrm{Au} \mathrm{NW}\left(\rho_{\mathrm{Au}}=6 \mu \Omega \mathrm{cm}\right)$ and by Mayadas for Al thin films $\left(\rho_{\mathrm{Al}}=9.5 \mu \Omega \mathrm{cm}\right) .{ }^{44}$ Given that the resistivities of the NWs are very close to the film values compared to the bulk values, we can infer that the thickness of the NWs (Al, 30-60 nm and $\mathrm{Au}, 45-20)$ is the main factor increasing the resistivity of the NWs with respect to bulk values either by surface or grain boundary scattering. However, it is interesting that in the size range of the NWs studied, we did not observe a dependence of the resistivity on the cross section area (width or thickness) as expected from size reduction of the NWs. This was also observed by Durkan et al. for Au wires that were not annealed as in our case. $^{43,45}$ If this dependence exists, it might be too small to be detected in our experimental conditions and dimensions. In our NWs we did not observe a change in grain size with the width or thickness of the NWs either. Given that the electrical resistivity and the grain size do not change with the size of the structures, this could be an indication that the resistivity of our NWs is dominated by grain boundary scattering. This is also supported by the fact that the grain sizes were smaller than the size of the NWs. Previous works reported that for thin films and NWs fabricated with templates or lift-off, the grain size was roughly equal to the structure dimensions. ${ }^{43,46,47}$ In our case we did not observe such relation and this could be related to the way structures are deposited in SL. Compared to lift-off or template growth, during SL the material lands freely on the surface without any lateral barrier restricting the spreading of the material or the growth of the structure. This probably affects the geometry and grain structure of the deposited NWs and this would have an impact on the electrical resistivity. We also observed that the increase in resistivity of NWs and films compared to bulk values is larger for Al than for Au. This behavior could be due to a larger surface roughness and a larger reflection at grain boundary scattering for $\mathrm{Al}$ than for $\mathrm{Au}$. The difference in the growth, shape, and grain structure of NWs fabricated by SL compare to lift-off or template methods may offer new information on the effect of the size and grain structure on the electrical conductivity of nanostructures. (See Supporting Information, Figure SI 6, for a comparison of the resistivity of the NWs with $\mathrm{Au}$ and $\mathrm{Al}$ thin films and Tables SI 1 and 2, for estimations of the resistivity of the NWs due to surface and the grain boundary scattering)

In conclusion, we have demonstrated the fabrication of metallic nanowires $<100 \mathrm{~nm}$ on a full wafer scale by stencil lithography without any resist processing. The nanowires are wider than the stencil apertures by tens of nanometers and the thickness decreases as the width of the stencil apertures decreases. The NWs fabricated by SL show an ohmic behavior, a higher than bulk resistivity and maximum current density in the order of $10^{8} \mathrm{~A} / \mathrm{cm}^{2}$. This opens an alternative to fabricate NWs in other substrates that are not compatible with resist-based lithography. Since the NWs fabricated by SL do not use any template, resist, or etching during their deposition, they can offer new properties and insight into the deposition and properties of metallic nanostructures.

Acknowledgment. This effort is sponsored by the Swiss Federal Office for Science and Education (OFES) in the framework of the EC-funded FP6 project NaPa (NMP4-CT2003-500120), the Swiss National Science Foundation (Project IC-Nano, 200021-112291/1), and the EPFL-STI Seed Funding. The authors are very grateful to the EPFL Centre of MicroNano-Technology (CMI) and its staff for their effort implementing stencil lithography, especially to Dr. Philippe Fluckiger, Dr. Philippe Langlet, Guy Clerc; and Dr. Georges-André Racine for their effort implementing stencil lithography at CMI. We are also grateful to Dr. Chan Woo Park and Dr. Schahrazede Mouaziz for their help and discussions, and to our colleagues at the Microsystems Laboratory for their very valuable support and help.

Supporting Information Available: Stencil wafers (100 mm diameter), stencil fabrication, corrugated membranes; $\mathrm{Au}$ NWs thickness reduction; grain structure and roughness of NWs; $\mathrm{Al}$ and $\mathrm{Au}$ NWs from I/V curves in Figure 2; $\mathrm{Al}$ and $\mathrm{Au}$ thin film resistivity; and an estimation of electrical resistivity of NWs due to surface and grain boundary scattering. This material is available free of charge via the Internet at http://pubs.acs.org.

\section{References}

(1) Lieber, C. M.; Wang, Z. L. MRS Bulletin 2007, 32 (2), 99-108.

(2) Cui, Y.; Lieber, C. M. J. Science 2001, 291 (5505), 851-853. 
(3) Hayashi, M.; Thomas, L.; Moriya, R.; Rettner, C.; Parkin, S. S. P. Science 2008, 320 (5873), 209-211.

(4) Boukai, A. I.; Bunimovich, Y.; Tahir-Kheli, J.; Yu, J.-K.; Goddard, W. A., III; Heath, J. R. Nature 2008, 451 (7175), 168-171.

(5) Hochbaum, A. I.; Chen, R.; Delgado, R. D.; Liang, W.; Garnett, E. C.; Najarian, M.; Majumdar, A.; Yang, P. Nature 2008, 451 (7175), 163167.

(6) Li, M.; Bhiladvala, R. B.; Morrow, T. J.; Sioss, J. A.; Lew, K.-K.; Redwing, J. M.; Keating, C. D.; Mayer, T. S. Nat. Nanotechnol. 2008, 3 (2), 88-92.

(7) Wang, J.; Gudiksen, M. S.; Duan, X.; Cui, Y.; Lieber, C. M. Science 2001, 293 (5534), 1455-1457.

(8) Wanekaya, A.; Chen, W.; Myung, N.; Mulchandani, A. Electroanalysis 2006, 18 (6), 533-550.

(9) Aravamudhan, S.; Kumar, A.; Mohapatra, S.; Bhansali, S. Biosens. Bioelectron. 2007, 22 (9-10), 2289-2294.

(10) Huang, S.; Chen, Y. Nano Lett. 2008, 8 (9), 2829-2833.

(11) McCord, M. A.; Rooks, M. J. Electron Beam Lithography. In SPIE Handbook of Microlithography, Micromachining and Microfabrication; Rai-Choudhury, P., Ed.; SPIE: Bellingham, WA, 1997; Vol. 1.

(12) Reyntjens, S.; Puers, R. J. Micromech. Microeng. 2001, (4), 287.

(13) Silverman, P. J. Intel Technol. J. 2002, 06 (2), 55-61.

(14) Chou, S. Y.; Krauss, P. R.; Renstrom, P. J. Science 1996, 272 (5258), $85-87$.

(15) Chou, S., Y.; Krauss, P., R.; Zhang, W.; Gou, L.; Zhuang, L. J. Vac. Sci. Technol., B 1997, 15 (6), 2897-2904.

(16) Deshmukh, M. M.; Ralph, D. C.; Thomas, M.; Silcox, J. Appl. Phys. Lett. 1999, 75 (11), 1631-1633.

(17) Villanueva, G.; Vazquez-Mena, O.; van den Boogaart, M. A. F.; Sidler, K.; Pataky, K.; Savu, V.; Brugger, J. Microelectron. Eng. 2008, 85 (5-6), 1010-1014.

(18) Dumas, C.; Grisolia, J.; Ressier, L.; Arbouet, A.; Paillard, V.; Ben Assayag, G.; Claverie, A.; van den Boogaart, M. A. F.; Brugger, J. Phys. Status Solidi A 2007, 204 (2), 487-491.

(19) Yan, X. M.; Contreras, A. M.; Koebel, M. M.; Liddle, J. A.; Somorjai, G. A. Nano Lett. 2005, 5 (6), 1129-1134.

(20) Brugger, J.; Berenschot, J. W.; Kuiper, S.; Nijdam, W.; Otter, B.; Elwenspoek, M. Microelectron. Eng. 2000, 53 (1-4), 403-405.

(21) Schlittler, R. R.; Seo, J. W.; Gimzewski, J. K.; Durkan, C.; Saifullah, M. S. M.; Welland, M. E. Science 2001, 292 (5519), 1136-1139.

(22) Lee, J. W.; Ju, B. K.; Jang, J.; Yoon, Y. S.; Kim, J. K. J. Mater. Sci. 2007, 42 (3), 1026-1030.

(23) Cojocaru, C.-V.; Harnagea, C.; Rosei, F.; Pignolet, A.; van den Boogaart, M. A. F.; Brugger, J. Appl. Phys. Lett. 2005, 86 (18), 183107.

(24) Rodriguez, A. F.; Heyderman, L. J.; Nolting, F.; Hoffmann, A.; Pearson, J. E.; Doeswijk, L. M.; van den Boogaart, M. A. F.; Brugger, J. Appl. Phys. Lett. 2006, 89 (14), 142508.
(25) Speets, E. A.; Riele, P. T.; van den Boogaart, M. A. F.; Doeswijk, L. M.; Ravoo, B. J.; Rijnders, G.; Brugger, J.; Reinhoudt, D. N.; Blank, D. H. A. Adv. Funct. Mater. 2006, 16 (10), 1337-1342.

(26) Zhou, Y. X.; Johnson, A. T.; Hone, J.; Smith, W. F. Nano Lett. 2003, 3 (10), 1371-1374.

(27) Sidler, K.; Vazquez-Mena, O.; Savu, V.; Villanueva, G.; van den Boogaart, M. A. F.; Brugger, J. Microelectron. Eng. 2008, 85 (5-6), 1108-1111.

(28) Arcamone, J.; van den Boogaart, M. A. F.; Serra-Graells, F.; Hansen, S.; Brugger, J.; Torres, F.; Abadal, G.; Barniol, N.; Perez-Murano, F. 2006 International Electron Devices Meeting, Vols 1 and 2 2006, 250253

(29) Vázquez-Mena, O.; Villanueva, G.; van den Boogaart, M. A. F.; Savu, V.; Brugger, J. Microelectron. Eng. 2008, 85 (5-6), 1237-1240.

(30) Tun, T. N.; Lwin, M.; Kim, A. H. H.; Chandrasekhar, A. N.; Joachim, C. Nanotechnology 2007, 18 (33), 335301.

(31) van den Boogaart, M. A. F.; Lishchynska, M.; Doeswijk, L. M.; Greer, J. C.; Brugger, J. Sens. Actuators, A 2006, 13 (0-131), 568-574.

(32) Kim, G. M.; van den Boogaart, M. A. F.; Brugger, J. Microelectron. Eng. 2003, 6 (7-68), 609-614.

(33) Lishchynska, M.; Bourenkov, V.; van den Boogaart, M. A. F.; Doeswijk, L.; Brugger, J.; Greer, J. C. Microelectron. Eng. 2007, 84 (1), 42-53.

(34) Horcas, I.; Fernandez, R.; Gomez-Rodriguez, J. M.; Colchero, J.; Gomez-Herrero, J.; Baro, A. M. Rev. Sci. Instrum. 2007, 78 (1), 013705 .

(35) Egger, S.; Ilie, A.; Fu, Y.; Chongsathien, J.; Kang, D.-J.; Welland, M. E. Nano Lett. 2005, 5 (1), 15-20.

(36) Kohler, J.; Albrecht, M.; Musil, C. R.; Bucher, E. Physica E 1999, 4 (3), 196-200.

(37) Racz, Z.; Seabaugh, A. J. Vac. Sci. Technol., B 2007, 25 (3), 857861.

(38) Lide, D., CRC Handbook of Chemistry and Physics, 87th ed.; Lide, D.R., Ed.; CRC Press: Boca Raton, FL, 2006.

(39) Ashcroft, N. W.; Mermin, N. D. Solid State Physics; Saunders College Publishing; Fort Worth, TX, 1976.

(40) Fuchs, K. Proc. Cambridge Philos. Soc. 1938, 34, 100-108.

(41) Sondheimer, E. H. Adv. Phys. 1952, 1 (1), 1-42.

(42) Mayadas, A. F.; Shatzkes, M. Phys. Rev. B 1970, 1 (4), 1382-1389.

(43) Durkan, C.; Welland, M. E. Phys. Rev. B 2000, 61 (20), 14215-14218.

(44) Mayadas, A. F. J. Appl. Phys. 1968, 39 (9), 4241-4245.

(45) Durkan, C.; Schneider, M. A.; Welland, M. E. J. Appl. Phys. 1999, 86 (3), 1280-1286.

(46) Mayadas, A. F.; Feder, R.; Rosenberg, R. J. Vac. Sci. Technol. 1969, 6 (4), 690-693.

(47) Steinhögl, W.; Schindler, G.; Steinlesberger, G.; Engelhardt, M. Phys. Rev. B 2002, 66 (7), 075414.

NL801778T 